\title{
Spatial resolution on a small animal RPC-PET prototype operating under magnetic field
}

A. Blanco $^{\mathrm{a}, *}$, N. Carolino ${ }^{\mathrm{a}}$, C.M.B.A. Correia $^{\mathrm{b}}$, L. Fazendeiro ${ }^{\mathrm{a}}$, Nuno C. Ferreira ${ }^{\mathrm{c}}$, M.F. Ferreira

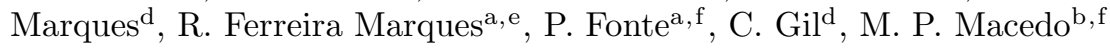

${ }^{a}$ LIP, Laboratório de Instrumentação e Física Experimental de Partículas, 3004-516 Coimbra, Portugal.

${ }^{\text {b} C E I, ~ C e n t r o ~ d e ~ E l e c t r o ́ n i c a ~ e ~ I n s t r u m e n t a c ̧ a ̃ o, ~ U n i v e r s i d a d e ~ d e ~ C o i m b r a, ~ 3004-516 ~ C o i m b r a, ~ P o r t u g a l . ~}$

' IBILI, Instituto Biomédico de Investigação de Luz e Imagem, Faculty of Medicine, 3000-548, Coimbra Portugal.

dICEMS, Departamento de Física, Universidade de Coimbra, 3004-516 Coimbra, Portugal.

eDepartamento de Física, Faculdade de Ciências e Tecnologia da Universidade de Coimbra, 3004-516 Coimbra, Portugal

${ }^{\mathrm{f}}$ ISEC, Instituto Superior de Engenharia de Coimbra, Quinta da Nora, 3030-199 Coimbra, Portugal.

It has been demonstrated in previous work that the RPC-PET technology is able to deliver radioisotope image resolutions approaching the physical limits of the PET principle. Here we study, by simulation, the effect of the magnetic field on the positron range to evaluate whether the spatial resolution of the RPC-PET could be improved by using an intense magnetic field. Six positron emitters of interest to small animal PET imaging $\left({ }^{18} \mathrm{~F},{ }^{11} \mathrm{C},{ }^{15} \mathrm{O}\right.$, ${ }^{68} \mathrm{G},{ }^{62} \mathrm{Cu}$ and ${ }^{86} \mathrm{Y}$ ) are considered. Results suggest that a three-fold improvement on the spatial resolution may be obtained under a magnetic field of $10 \mathrm{~T}$ for the higher energy radioisotopes like ${ }^{86} \mathrm{Y}$ or ${ }^{62} \mathrm{Cu}$, and by about $20 \%$ for the lower energy ones, like ${ }^{18} \mathrm{~F}$ or ${ }^{11} \mathrm{C}$.

(C) 2005 Elsevier Science. All rights reserved

PACS: 29.40.Cs; 87.59.Vb; 87.62.+n

Keywords: Gaseous detectors, RPC, PET, magnetic field;

\section{Introduction}

Positron emission tomography (PET) is one of the in vivo imaging modalities largely developed in the last decades. Its strength resides in the ability to accurately measure the amount of tracer accumulation in organs, merging the best combination of sensitivity and spatial resolution.

One of the PET applications is the imaging of small animals, particularly useful for testing hypothesis during the study of diseases, development of new imaging and therapeutic drugs and validations of gene therapies. In this modality, small animals, like transgenic mice and rats, are used as experimental models owing to their ge-

*Corresponding author. Tel.: (+351)239 833 465; fax: (+351) 239822 358; e-mail: alberto@coimbra.lip.pt. netic similitude with humans, short reproductive cycle and simple breeding. Due to the small dimensions of these animals dedicated high spatial resolution instruments are often required.

\subsection{RPC-PET}

Based in some preliminary simulation results [1], which suggest that a system built with RPCs may deliver sub-millimeter spatial resolution free of parallax error, capabilities very attractive for the imaging of small animals, a first prototype of a small animal PET has been designed and tested.

The prototype [2] is composed of two opposite counting heads at a distance of $60 \mathrm{~mm}$, each one composed of 16 stacked single gap RPC counters made from copper (cathode) and glass (anode) electrodes. Two coordinates of the interaction 
point of the photon are measured in this prototype: the tangential coordinate and the depth of interaction (DOI), allowing to obtain a 2D planar projection image.

By imaging point-like ${ }^{22} \mathrm{Na}$ positron sources, positioned in the transaxial plane at different positions along the tangential direction, a homogeneous image spatial resolution of $0.3 \mathrm{~mm}$ FWHM and $0.8 \mathrm{~mm}$ FWTM was obtained after reconstruction by an ML-EM type algorithm [3], demonstrating the parallax-free imaging capability of the system [4]. The different components that contribute to the spatial resolution were individually modeled and combined by convolution [5]. The resulting distribution was then adjusted to the observed sinogram profile, yielding estimates for some of the resolution components. The detector intrinsic spatial resolution was estimated to be $0.22 \mathrm{~mm}$ FWHM and a value of $3.75 \mathrm{~mm}^{-1}$ was measured for the parameter $\mathrm{k}_{2}$ (which represents the positron range distribution tails that dominate the imaging resolution), validating experimentally positron range simulations done by other authors [6]. The counter-intuitive way in which the positron range distribution is convoluted, due to its non-Gaussian shape, which invalidates the usual quadratic rule for the addition of errors, was also studied. A completely optimized system was simulated to evaluate the expected sensitivity and system count rate performance, suggesting a central point absolute sensitivity up to $21 \mathrm{cps} / \mathrm{kBq}$ and a NECR figure up to $320 \mathrm{kcps}$ at an activity of around $2.3 \mathrm{MBq} / \mathrm{cm}^{3}$ for a mouse size phantom $\left(38 \mathrm{~cm}^{3}\right)[5]$.

\subsection{Effects of a magnetic field}

The magnetic field exerts a Lorentz force on a charged particle in motion given by

$$
F_{l o r}=q V \times B
$$

were $V$ is the velocity vector, $B$ the magnetic field vector and $q$ the particle charge. Due to the nature of the cross-product, a positron moving at an angle to the axis of the magnetic field will describe a helical path. Thus, a magnetic field collinear with the axis of the scanner will improve its transaxial resolution, since the positron range is reduced in that plane (fig1), while keeping the axial resolution unaltered.

a)

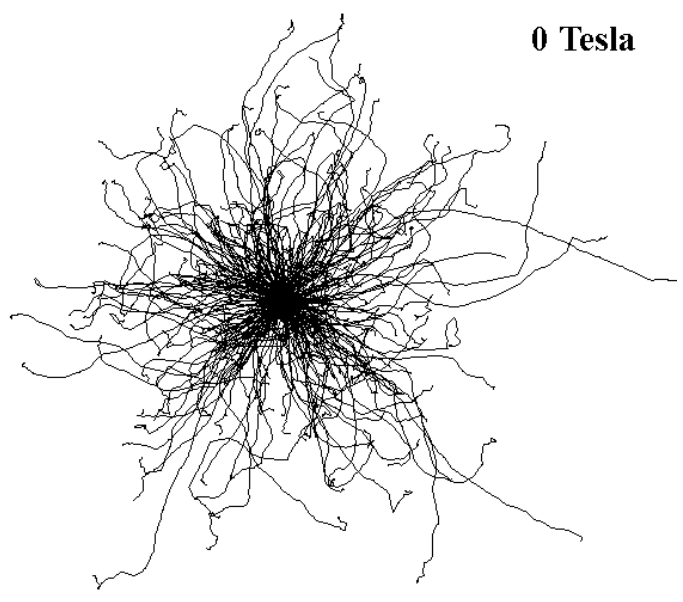

b)

10 Tesla

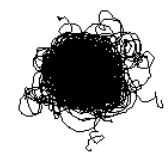

Y86 - $\mathrm{E}_{\max } 3.150 \mathrm{MeV}$

Figure 1. Influence of the magnetic field on positron range, for the radioisotope ${ }^{86} \mathrm{Y}$ $\left(\mathrm{E}_{\max }=3.15 \mathrm{MeV}\right)$ in water, illustrated by the GEANT4 simulation toolkit. a) 0 Tesla and b) 10 Tesla field orthogonal to the paper plan. The average distance between the emission and annihilation point is reduced in presence of the magnetic field.

The use of a magnetic field to increase the spatial resolution of a PET scanner has been studied by several authors $[7,8,9,10]$. However, all those studies were made for systems with spatial resolutions above $2-3 \mathrm{~mm}$, so the impact of using a magnetic field in a system with sub-millimeter resolution is not clear. 
The present work aims at investigating, by simulation, the possibility of improving the RPCPET spatial resolution by the use of a strong magnetic field. This possibility is especially interesting because the RPC technology is compatible with magnetic fields (its performance is not altered by the field), which would allow operation within a Magnetic Resonance Imager (MRI) [11].

\section{Methods}

The spatial resolution was calculated using the distribution described in [5], in which the different components of the spatial resolution were individually modeled and combined by convolution. The annihilation photon non-collinearity, detector effects and scattered background were fixed to the values used in [5], while the positron distributions for a point-like positron source were simulated for different magnetic field intensities.

The positron range simulation was performed using the simulation toolkit Geant4.05.02 with the low energy extension [12]. The Monte Carlo algorithm generates positrons in water at a fixed point with energy distributions corresponding to interesting positron sources in small animal PET imaging [13, 14, 15, 16]: ${ }^{18} \mathrm{~F} \quad\left(\mathrm{E}_{\max }=0.635 \mathrm{MeV}\right),{ }^{11} \mathrm{C}$ $\left(\mathrm{E}_{\max }=0.970 \mathrm{MeV}\right),{ }^{15} \mathrm{O} \quad\left(\mathrm{E}_{\max }=1.720 \mathrm{MeV}\right)$, ${ }^{68} \mathrm{G} \quad\left(\mathrm{E}_{\max }=1.900 \mathrm{MeV}\right),{ }^{62} \mathrm{Cu} \quad\left(\mathrm{E}_{\max }=2.910\right.$ $\mathrm{MeV}),{ }^{86} \mathrm{Y} \quad\left(\mathrm{E}_{\max }=3.150 \mathrm{MeV}\right)$. The resulting positron distributions were fitted with a double exponential $[5,17]$ and convoluted with the remaining contributions to obtain the overall spatial resolution.

\section{Results}

Figure 2 shows the positron range distributions for ${ }^{86} \mathrm{Y}$, for different magnetic fields, clearly showing a marked narrowing with increasing field strength.

Figure 3 shows the spatial resolution expected for RPC-PET for the six different radioisotopes under magnetic fields from 0 to 10 Tesla. In order to decrease the FWHM and FWTM values for the lower energy radioisotopes, ${ }^{18} \mathrm{~F}$ or ${ }^{11} \mathrm{C}$, by factors of $\sim 1.2$ and $\sim 1.6$, respectively, a magnetic field of $10 \mathrm{~T}$ is required. However the application of the same fields results in a significant decrease in the FWHM and FWTM values for the higher energy radioisotopes. Indeed, for ${ }^{86} \mathrm{Y}$ and ${ }^{62} \mathrm{Cu}$, improvement by factors of $\sim 1.8$ and $\sim 2.7$, respectively, are observed for $5 \mathrm{~T}$, whereas for $10 \mathrm{~T}$ these factors become $\sim 3.1$ and $\sim 4.9$.

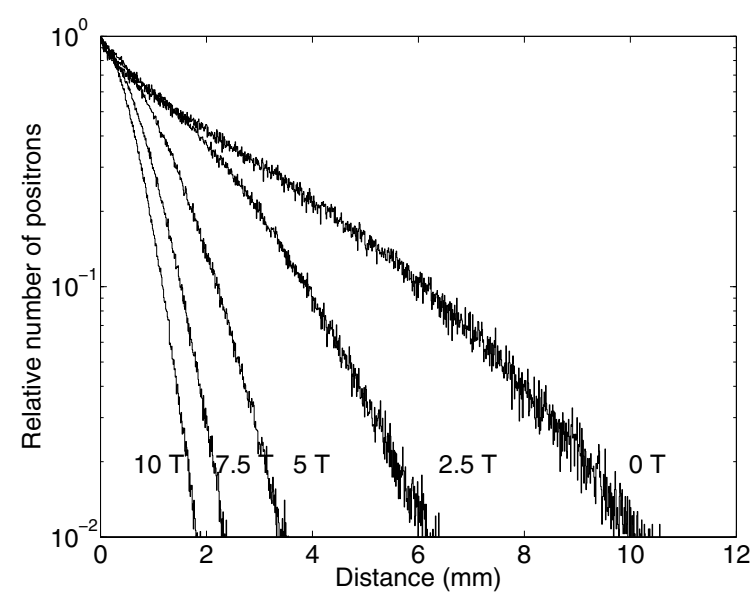

Figure 2. Geant4 simulation of positron range distributions for ${ }^{86} \mathrm{Y}\left(\mathrm{E}_{\max }=3.150 \mathrm{MeV}\right)$ for different magnetic intensities.

An experimental test was made using two permanent magnets mounted on an iron core, which created a field of $\sim 0.3 \mathrm{~T}$ over the point-like ${ }^{22} \mathrm{Na}$ source. As our system is able to see directly the positron range distribution tails [5], controlled by the $\mathrm{k}_{2}$ parameter, any modification on this distribution, produced by the magnetic field, should be clearly seen. Indeed, as expected for this field intensity, no modification of $\mathrm{k}_{2}$ was observed.

\section{Conclusion}

We have calculated the improvement in the spatial resolution of the RPC-PET prototype by the use of strong magnetic fields for six radioisotopes with interest in small animal PET imaging. 


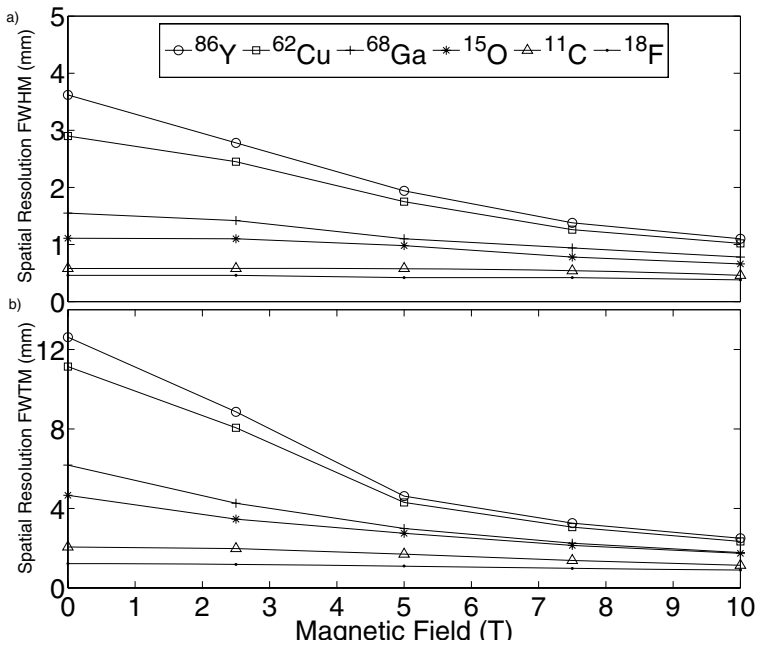

Figure 3. Calculated spatial resolution a) FWHM and b) FWTM for ${ }^{18} \mathrm{~F},{ }^{11} \mathrm{C},{ }^{15} \mathrm{O},{ }^{68} \mathrm{G},{ }^{62} \mathrm{Cu},{ }^{86} \mathrm{Y}$ as a function of the magnetic field, from 0 to 10 $\mathrm{T}$.

The spatial resolution was calculated from an experimental distribution in which the positron range contribution was calculated by simulation, using the Geant4 toolkit.

An improvement in the spatial resolution by a factor $\sim 3.1$ was obtained for high positron energy radioisotopes, ${ }^{86} \mathrm{Y}$ or ${ }^{62} \mathrm{Cu}$, and by a factor $\sim 1.2$ for lower energy radioisotopes, ${ }^{18} \mathrm{~F}$ or ${ }^{11} \mathrm{C}$, at a magnetic field of $10 \mathrm{~T}$.

The use of intense magnetic fields seems to be a viable approach to increase the spatial resolution of the RPC-PET, especially for high positron energy radioisotopes. In addition, since the RPCPET technology is compatible with Magnetic Resonance Imaging, a structural merging in view of the co-register of both types of information may be feasible.

\section{Acknowledgments}

The authors gratefully acknowledge L. Lopes for many useful discussions and the technical support of A. Pereira.

This work was financed by "Fundação para a Ciência e Tecnologia" and FEDER under contracts POCTI/FP/FNU/50171/2003 and POCI/FP/63411/2005.

\section{REFERENCES}

1. A. Blanco, et al., Nucl. Instr. and Meth. A 508 (2003) 88-93.

2. A. Blanco, et al., Nucl. Instr. and Meth. A 533 (2004) 139-143.

3. L. Fazendeiro, et al., proceedings of IEEE Medical Image Conference 2004, M2-177.

4. A.Blanco, et al., presented at International Conference on new Developments in Photodetection 2005, accepted for publication at Nucl. Instr. and Meth. A.

5. A. Blanco, et al., proceedings of IEEE Medical Image Conference 2004, M2-21, accepted for publication at IEEE Trans. Nucl. Sci.

6. Craig S. Levin, Edward J. Hoffman, Phys. Med. Biol. 44 (1999) 781-799.

7. Hammer et al. Phy. Med. Biol. 40 (1995) 691697.

8. Hammer et al., IEEE Trans. Nucl. Sci., vol 42 No 4, August 1995.

9. Raylman et al., IEEE Trans. Nucl. Sci., vol 43 No 4, August 1996.

10. Wirrwar et al., IEEE Trans. Nucl. Sci., vol 44 No 2, April 1997.

11. Townsend DW et all, Eur Radiol 2001; 11 (10): 1968-74.

12. G4 http://geant4.web.cern.ch/geant4/

13. Richard Laforest, et al. Eur J Nucl Med Mol Imaging (2005) 32,764-770.

14. Gambhir SS, Jour. Nucl. Med, 2001. 42, 1S92S.

15. Paul McQuade et al. J. Med. Chem. 2005, 48, 2985-2992.

16. Terry L. Sharp et all, Nucl. Med. Biol. 32 (2005) 875-84.

17. Stephen E. Derenzo, IEEE Trans. Nucl. Sci., vol 33, No. 1, February 1986, 565-569. 